\title{
Dynamics, chaos and synchronization of self-sustained electromechanical systems with clamped-free flexible arm
}

\author{
C.A. Kitio Kwuimy • P. Woafo
}

Received: 28 June 2007 / Accepted: 16 October 2007 / Published online: 8 November 2007

(C) Springer Science+Business Media B.V. 2007

\begin{abstract}
An electromechanical system with flexible arm is considered. The mechanical part is a linear flexible beam and the electrical part is a nonlinear selfsustained oscillator. Oscillatory solutions are obtained using an averaging method. Chaotic behavior is studied via the Lyapunov exponent. The synchronization of regular and chaotic states of two such devices is discussed and the stability boundaries for the synchronization process are derived using the Floquet theory. We compare the results obtained from a finite difference simulation to those from the classical modal approach.
\end{abstract}

Keywords Electromechanical devices · Flexible arms · Oscillatory and chaotic states .

Synchronization

C.A. Kitio Kwuimy · P. Woafo ( $\square)$

Laboratory of Modeling and Simulation in Engineering and Biological Physics, University of Yaounde I, Box 812, Yaounde, Cameroon

e-mail: pwoafo1@yahoo.fr

P. Woafo

Georg Forster Research Fellow, Max-Planck Institute for Dynamics and Self-Organisation, Bunsenstr. 10, 37073

Gottingen, Germany

\section{Introduction}

Recently, various studies have been devoted to nonlinear electromechanical devices consisting of a nonlinear electric circuit coupled magnetically or electrostatically to rigid arm [1-9]. These devices are described by two coupled nonlinear differential equations. The particular interest to these devices is that they are inherently present in everyday life both at the domestic and industrial levels for the automation of various processes [4]. This is, for example, the case of multifrequency or chaotic industrial shaker.

Amongst these systems, self-sustained devices are particularly interesting since they can run without external excitation. Reference [1] considers an electrical implementation of a Van der Pol oscillator driving a rigid arm, while the case of a Rayleigh electrical oscillator is studied in [2]. The case of a self-sustained electromechanical system with flexible arm and nonlinear coupling is investigated in [3]. These studies revealed that such devices can present complex phenomena (chaos, hysteresis and jump phenomena). An important problem with these nonlinear phenomena is that two identical systems launched with initial conditions belonging to different basins of attraction will finally circulate on different orbits. For engineering applications, it is sometimes of particular interest to have various robot arms acting in a synchronized manner. For instance, for industrial shakers and mixers, the increase of the production rate and precision requires a network of arms working in a synchronized 
way. Synchronization phenomenon in nonlinear science has seen a growing interest this last decade. The problem of frequency synchronization of two excited pendula with dissipative and elastic (linear) coupling is considered in [10], an adaptive algorithm for the synchronization of two different chaotic electromechanical systems is presented in [11]. The question of master-slave synchronization of two identical systems was one of the goals in [2], an extension to a ring of such devices is investigated in [8]. The case of a system with multiple outputs is studied in [7]. In [12, 13], the case of delay (autonomous and nonautonomous) systems is studied.

The aim of this paper is to extend the above studies to an electromechanical device with cantilever arm. This constitutes a new mathematical and numerical challenge. Moreover, this is a new interesting area of applications since many industrial tasks are carried out through flexible structures. The device under consideration here consists of a Rayleigh-Duffing electrical circuit coupled magnetically to a clamped-free flexible beam.

The paper is organized as follows. Section 2 consists of three parts. The first part presents the nonlinear electromechanical device as well as the resulting partial differential equations. The second part considers the one-mode approximation of the beam dynamics to derive a set of two nonlinear differential equations for the amplitudes of the first mode and electric charge of the capacitor. These equations constitute the basis of the analytical and the semi-analytical investigation. The third part of Sect. 2 deals with the presentation of the finite difference algorithm for the direct numerical simulation of the full equations of the electromechanical device. In Sect. 3, the averaging method is used to derive the approximate oscillatory states whose amplitudes are compared to the results of the numerical simulation. Section 4 is devoted to the question of chaotic behavior while Sect. 5 uses the unidirectional coupling scheme to find the good parameters leading to the synchronization of a second similar device (slave device) to the motion of the first device called master. This is done both in the case of periodic oscillatory and chaotic behavior. The conclusion is given in Sect. 6 .

\section{Model, equations and numerical scheme}

\subsection{Model}

The model shown in Fig. 1 is an electrical oscillator coupled through a magnet to a clamped-free flexible beam. The electrical part consists of a nonlinear resistor (NLR), a nonlinear condenser (NLC), C and an inductor $\mathrm{L}$, all connected in series. Two types of nonlinear components are considered in the model. The voltage of the condenser is a nonlinear function of the instantaneous electrical charge and is expressed by

$V_{C}=\frac{1}{C_{0}} q_{0}+a_{3} q_{0}^{3}$

where $C_{0}$ is the linear value of $C$ and $a_{3}$ is a nonlinear coefficient depending on the type of the capacitor [14]. The current-voltage characteristics of the resistor is defined as

$V_{R}=-R_{0} i_{0}\left(\frac{i}{i_{0}}-\frac{1}{3}\left(\frac{i}{i_{0}}\right)^{3}\right)$

where $R_{0}$ and $i_{0}$ are, respectively, the characteristics resistance and current; $i$ is the current through the resistor. This nonlinear resistor can be realized using a block consisting of two transistors [15] or a series of diodes [16]. With this resistor, the system has the property to exhibit self-excited oscillations. The currentvoltage characteristics of the linear inductor is

$V_{L}=L \frac{d i}{d \tau}$

where $\tau$ is the time.

The mechanical part is a flexible beam of length $l_{0}$. The beam is presumed to be a slender, isotropic, uniform rod. It is fixed at its top and free at the base. The magnetic coupling between both parts is made at a point $X_{1}$. It creates the Laplace force in the mechanical part and the Lenz electromotive voltage in the electrical part. Using the electrical and mechanical laws, it is found that the model is described by the following equations

$$
\begin{aligned}
& L \frac{d^{2} q_{0}}{d \tau^{2}}-R_{0}\left(1-\frac{1}{3 i_{0}^{2}}\left(\frac{d q_{0}}{d \tau}\right)^{2}\right) \frac{d q_{0}}{d \tau}+\frac{q_{0}}{C_{0}}+a_{3} q_{0}^{3} \\
& \quad=-B_{f} l \frac{\partial W}{\partial \tau} \delta\left(X-X_{1}\right),
\end{aligned}
$$


Fig. 1 An

electromechanical transducer with

clamped-free flexible arm

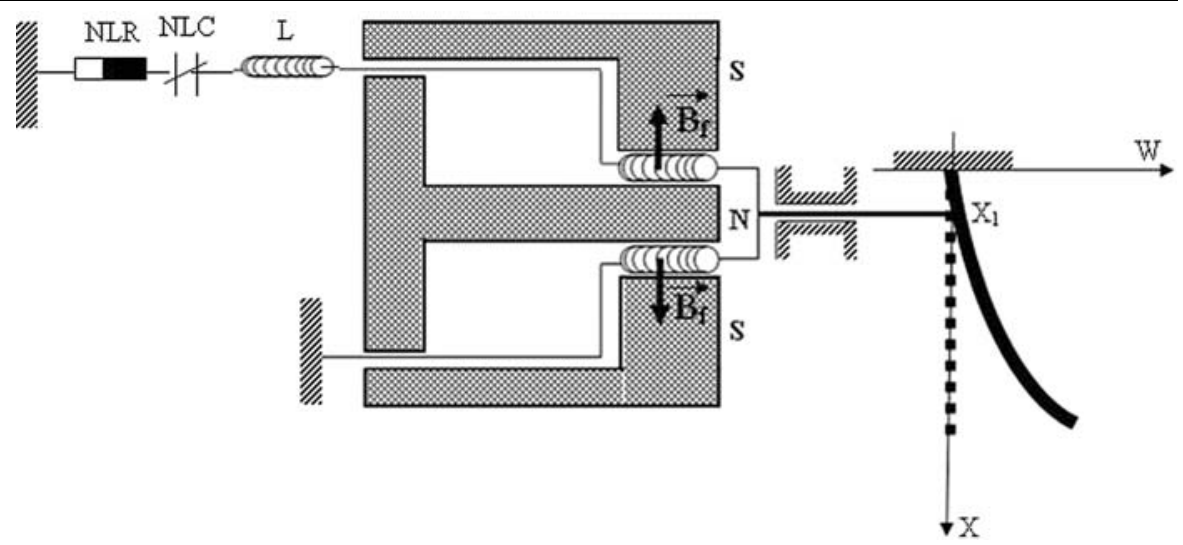

The beam boundary conditions are given as follows

$$
W(0, \tau)=0 ; \quad\left(\frac{\partial W}{\partial X}\right)(0, \tau)=0,
$$

$\forall \tau \in \mathbb{R}_{+}$, at the clamped end,

$\left(\frac{\partial^{2} W}{\partial X^{2}}\right)\left(l_{0}, \tau\right)=0$

$\left(\frac{\partial^{3} W}{\partial X^{3}}\right)\left(l_{0}, \tau\right)=0, \quad \forall \tau \in \mathbb{R}_{+}$, at the free end.

$E$ is the Young modulus of the beam, $\rho$ is the beam density, $S$ and $I$ are respectively the area and the moment of inertia of the beam cross section. $W(X, \tau)$ is the transversal deflection of the beam, $X$ is the spatial coordinate, $\lambda$ is the mechanical damping coefficient which is assumed to be constant, $B_{f}$ is the intensity of the magnetic field and $l$ is the length of the current wire in the coupling domain. $\delta($.) stands for the Dirac delta function; it expresses the fact that the coupling is made at a point $X_{1}$ of the flexible beam.

We introduce the dimensionless variables

$$
t=\omega_{1} \tau, \quad v=\frac{W}{l_{0}}, \quad x=\frac{X}{l_{0}}, \quad q_{0}=Q q,
$$

where $\omega_{1}=(1.875)^{2} \mathrm{rad} / \mathrm{s}$ and $Q=\frac{i_{0}}{\omega_{1}} \sqrt{3}$. Consequently, (4) and (5) become

$$
\begin{aligned}
& \frac{d^{2} q}{d t^{2}}-\varepsilon_{1}\left(1-\left(\frac{d q}{d t}\right)^{2}\right) \frac{d q}{d t}+w_{0}^{2} q+b q^{3} \\
& =-f_{2} \frac{\partial v}{\partial t} \delta\left(x-x_{1}\right),
\end{aligned}
$$

$\frac{\partial^{2} v}{\partial t^{2}}+\varepsilon_{2} \frac{\partial v}{\partial t}+a^{2} \frac{\partial^{4} v}{\partial x^{4}}=f_{1} \frac{d q}{d t} \delta\left(x-x_{1}\right)$,

with

$\varepsilon_{1}=\frac{R_{0}}{L \omega_{1}}, \quad \omega_{0}^{2}=\frac{1}{L C_{0} \omega_{1}^{2}}, \quad b=\frac{a_{3} Q^{2}}{L \omega 1^{2}}$,

$f_{2}=\frac{B_{f} l_{0} l}{L \omega_{1} Q}$,

$\varepsilon_{2}=\frac{\lambda}{\rho S \omega_{1}}, \quad a^{2}=\frac{E I}{\rho S l_{0}^{4} \omega_{1}^{2}}, \quad f_{1}=\frac{B_{f} l l_{0}}{L \omega_{1} Q}$

and the boundary conditions (6) and (7) become

$v(0, t)=0, \quad \frac{\partial v}{\partial x}(0, t)=0$,

$\forall t \in \mathbb{R}_{+}$, at the clamped end,

$\frac{\partial^{2} v}{\partial x^{2}}(1, t)=0, \quad \frac{\partial^{3} v}{\partial x^{3}}(1, t)=0$,

$\forall t \in \mathbb{R}_{+}$, at the free end.

\subsection{Mode equations}

For the analytical investigation, it is convenient to assume an expansion of the deflection $v(x, t)$ in terms of the combination of linear free oscillation modes. Due 
to the complexity of the eigenfunctions of the beam fixed at one end and free at the other, we will consider in the analytical treatment only the first mode. Thus, we can write

$v(x, t)=y_{1}(t) \phi_{1}(x)$

where

$$
\begin{aligned}
\phi_{1}(x)= & \cos \left(k_{1} x\right)-\cosh \left(k_{1} x\right) \\
& -\frac{\cos \left(k_{1}\right)+\cosh \left(k_{1}\right)}{\sin \left(k_{1}\right)+\sinh \left(k_{1}\right)} \\
& \times\left[\sin \left(k_{1} x\right)-\sinh \left(k_{1} x\right)\right] .
\end{aligned}
$$

The expression of $\phi_{1}(x)$ can be found in classic books on beam dynamics such as [17]. The eigenvalue $k_{m}$ for the mode $m$ is obtained from the transcendental equation

$\cos \left(k_{m}\right) \cosh \left(k_{m}\right)+1=0$.

This equation gives $k_{1} \approx 1.875$.

Inserting (13) into (9) and (10), multiplying (10) by $\phi_{1}(x)$, integrating over the nondimensional length of the beam and using the orthogonality of eigenfunctions, we obtain

$$
\begin{aligned}
& \frac{d^{2} q}{d t^{2}}-\varepsilon_{1}\left(1-\left(\frac{d q}{d t}\right)^{2}\right) \frac{d q}{d t}+w_{0}^{2} q+b q^{3} \\
& =-f_{21} \frac{d y_{1}}{d t} \\
& \frac{d^{2} y_{1}}{d t^{2}}+\varepsilon_{2} \frac{d y_{1}}{d t}+w_{01}^{2} y_{1}=f_{11} \frac{d q}{d t}
\end{aligned}
$$

with

$f_{11}=f_{1} \phi_{1}\left(x_{1}\right), \quad f_{21}=f_{2} \phi_{1}\left(x_{1}\right)$,

$w_{01}^{2}=w_{1}^{2} a^{2}$.

Thus, the one-mode dynamics is described by a Rayleigh-Duffing oscillator coupled to a linear harmonic oscillator equation. A linear stability analysis of the fixed stationary point $\left(q=0, \frac{d q}{d t}=0, y_{1}=0\right.$, $\frac{d y_{1}}{d t}=0$ ) shows that it is stable for $\varepsilon_{1}<\varepsilon_{2}<\frac{f_{11} f_{21}}{\varepsilon_{1}}$.

\subsection{The finite difference algorithm}

For obtaining a numerical solution of (9) and (10), we use the finite difference scheme. In this respect, we divide the nondimensional beam length in $n$ intervals of length $h_{x}$, e.g., $h_{x}=\frac{1}{n}$. Also, the time is discretized in units of length $h_{t}$. Therefore, one can write $x_{i}=(i-1) h_{x}$ and $t_{j}=j h_{t}$ where $i$ and $j$ are integer variables. Consequently, (9) and (10) become

$$
\begin{aligned}
& \frac{d^{2} q}{d t^{2}}-\varepsilon_{1} \frac{d q}{d t}\left(1-\left(\frac{d q}{d t}\right)^{2}\right)+w_{0}^{2} q+b q^{3} \\
& =-f_{2} \frac{v_{i, j+1}-v_{i, j}}{h_{t}} \delta_{i-1, i_{x_{1}}} \\
& A_{1} v_{i, j+1}+A_{2} v_{i, j}+A_{3} v_{i, j-1}+A_{4}\left(v_{i+2, j}+v_{i-2, j}\right) \\
& \quad+A_{5}\left(v_{i+1, j}+v_{i-1, j}\right)=f_{1} \frac{d q}{d t} \delta_{i-1, i_{x_{1}}}
\end{aligned}
$$

for $i=2, \ldots, n+1$ and $\forall j \in \mathbf{N}$, with

$$
\begin{aligned}
A_{1} & =\frac{1}{h_{t}^{2}}+\frac{\varepsilon_{2}}{2 h_{t}}, & A_{2} & =\frac{-2}{h_{t}^{2}}+\frac{6 a}{h_{x}^{4}}, \\
A_{3} & =\frac{1}{h_{t}^{2}}-\frac{\varepsilon_{2}}{2 h_{t}}, & A_{4} & =\frac{a^{2}}{h_{x}^{4}},
\end{aligned}
$$

The boundary conditions are $(\forall j \in \mathbf{N})$

$v_{1, j}=0, \quad v_{0, j}=v_{2, j}, \quad$ at the clamped end, (20)

$v_{n+2, j}=2 v_{n+1, j}-v_{n, j}$,

$v_{n+3, j}=v_{n-1, j}+2 v_{n+2, j}-2 v_{n, j}, \quad$ at the free end.

One can show that the discretization scheme is stable if

$\frac{8}{h_{x}^{4}} \leq \frac{1}{h_{t}^{2}}\left[1+\sqrt{1-\frac{\left(\varepsilon_{2} h_{t}\right)^{2}}{4}}\right]$

with $\varepsilon_{2} h_{t} \leq 2$.

\section{Oscillatory states}

Oscillatory solutions of (16) and (17) are obtained by using the Krylov-Bogoliubov averaging method described in [18, 19]. In this line, we set $q=A \sin \left(\omega_{0} t+\right.$ $\left.\varphi_{1}\right), y_{1}=B \sin \left(\omega_{01} t+\varphi_{2}\right)$. The amplitudes $A$ and $B$ 
satisfy the following set of first order differential equations

$\frac{d A}{d t}=\frac{\varepsilon_{1} A}{2}\left(1-\frac{3}{4} A^{2} w_{0}^{2}\right)-\frac{f_{21} B w_{01}}{2 w_{0}} \cos (\varphi)$,

$\frac{d B}{d t}=-\frac{\varepsilon_{2} B}{2}+\frac{f_{11} A w_{0}}{2 w_{01}} \cos (\varphi)$,

$\frac{d \varphi}{d t}=-\frac{3 b A^{2}}{8 w_{0}}+\left[\frac{f_{21} B w_{01}}{2 A w_{0}}-\frac{f_{11} A w_{0}}{2 B w_{01}}\right] \sin (\varphi)$

with $\varphi=\varphi_{1}-\varphi_{2}$. For the steady-states solutions, we obtain

$c_{6} A^{6}+c_{4} A^{4}+c_{2} A^{2}+c_{0}=0$

$B^{2}=M A^{2}\left(4-3 A^{2} w_{0}^{2}\right)$,

where

$$
\begin{aligned}
& c_{6}=27 \mu \nu^{2} w_{0}^{6}+3 \chi w_{0}^{2}, \\
& c_{4}=18 \chi \nu w_{0}^{4}(1-4 \eta)-4 \chi+9 v^{2} w_{0}^{4}(1-4 \mu), \\
& c_{2}=6 \nu w_{0}^{2}(1-4 v)(1-4 \mu)+3 \mu w_{0}^{2}(1-4 \nu)^{2}, \\
& c_{0}=(1-4 \mu)(1-4 \nu)^{2}, \quad \nu=\frac{\varepsilon_{1}}{4 \varepsilon_{2}}, \\
& \mu=\frac{\varepsilon_{1} \varepsilon_{2}}{4 f_{1 n} f_{2 n}}, \\
& \kappa=\frac{\varepsilon_{1}}{\varepsilon_{2} f_{1 n} f_{2 n} w_{0}^{2}}, \quad \chi=\frac{9 b^{2} \kappa}{64}, \\
& M=\frac{\varepsilon_{1} w_{0}^{2} f_{11}}{4 \varepsilon_{2} w_{0 n}^{2} f_{21}} .
\end{aligned}
$$

Let us note that there is a trivial steady-state defined by $A_{0}=B_{0}=0$. Equations (26) and (27) are solved using the Newton-Raphson algorithm.

Figures 2 and 3 show the amplitude curves of the beam at its free end and the charge of condenser in terms of the mechanical dissipative coefficient $\varepsilon_{2}$ for two different sets of values of parameters of the system. The numerical simulation results of (16) and (17) and those of (18) and (19) are also reported in the same figures. The numerical results of (16) and (17) are called semi-analytical ones. For Fig. 2, the analytical and semi-analytical curves show a complete quenching phenomena of oscillation in the region $\varepsilon_{1}<\varepsilon_{2}<\frac{f_{11} f_{21}}{\varepsilon_{1}}$. This result was also obtained in $[1,2]$ for a self-sustained oscillator coupled to a rigid rod. With this choice of values, the numerical

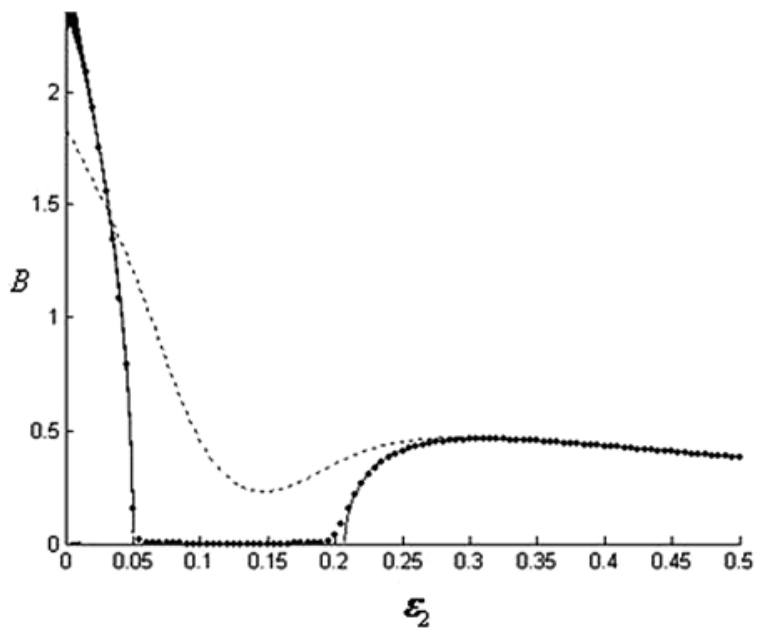

(a)

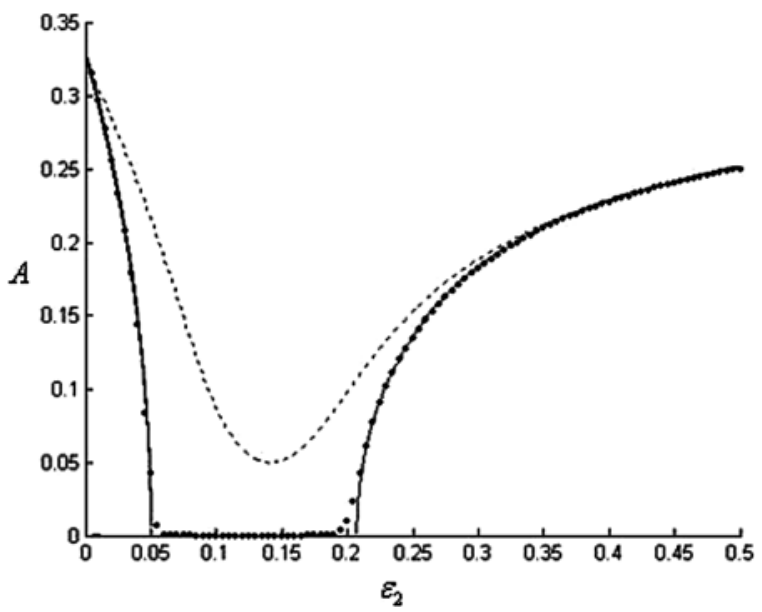

(b)

Fig. 2 Amplitudes of the mechanical part (a) and electrical part (b) as function of beam dissipation coefficient. Analytical curve (lines); semi-analytical curve (points); numerical curve (dash lines) with $b=0.1, a=1, w_{01}=w_{0}=w_{1} a, \varepsilon_{1}=0.05$, $f_{1}=1.4, f_{2}=0.1$

curves (those from (18) and (19)) do not corroborate this result. This is due to the fact that for the analytical and semi-analytical treatment, only one mode (the first) was taken into account. We observe that the effects of other modes, in spite of the fact that we are at the perfect resonance, cannot always be neglected. Making another choice of values of the parameters, we obtain quenching phenomena also with the partial differential equation (Fig. 3) for $0.032<\varepsilon_{2}<0.53$, while with the semi-analytical treatment, this occurs for $0.01<\varepsilon_{2}<0.73$. This corresponds to the stability 


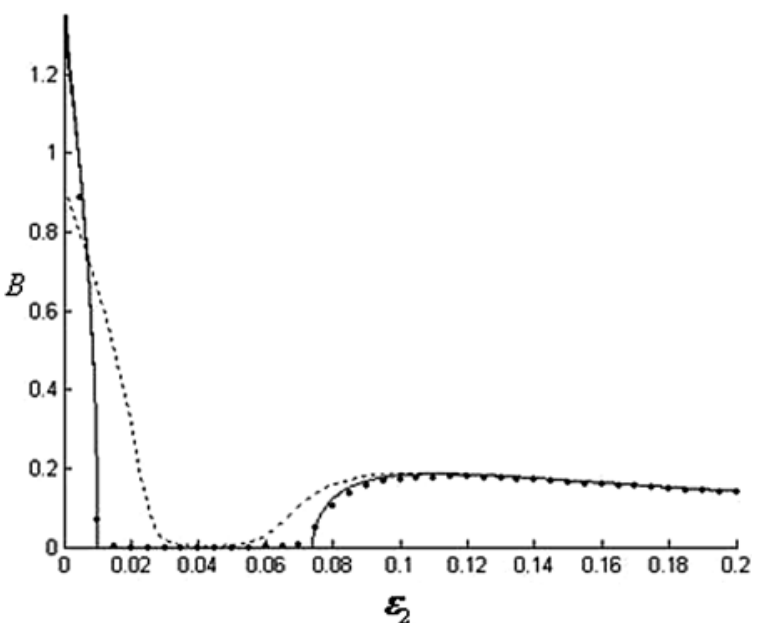

(a)

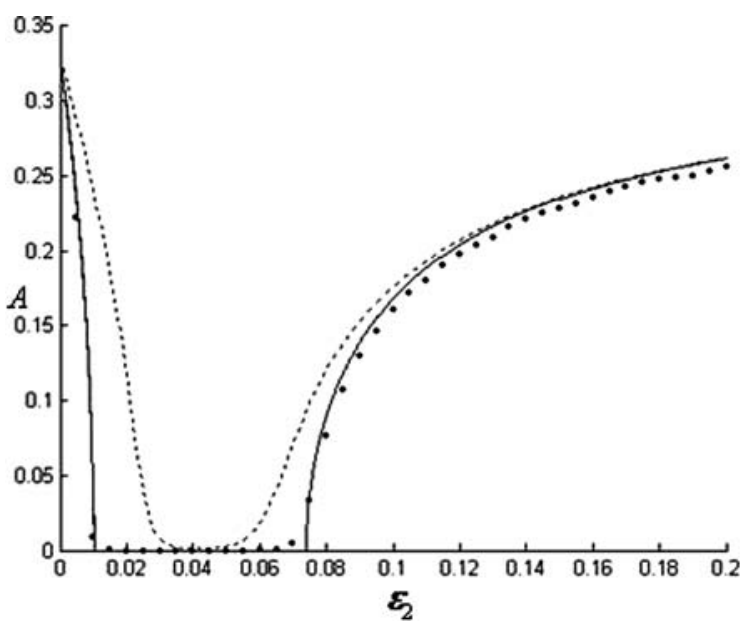

(b)

Fig. 3 Amplitudes of the mechanical part (a) and electrical part (b) as function of beam dissipation coefficient. Analytical curve (lines); semi-analytical curve (points); numerical curve (dash lines) with $b=0.01 ; a=1 ; w_{01}=w_{0}=w_{1} a ; \varepsilon_{1}=0.01$; $f_{1}=0.2 ; f_{2}=0.05$

interval $\varepsilon_{1}<\varepsilon_{2}<\frac{f_{11}}{f_{21}}$ of the stationary point ( $q=0$, $\frac{d q}{d t}=0, y_{1}=0, \frac{d y_{1}}{d t}=0$ ).

\section{Chaotic behavior}

In this section, we find how chaos arises in our device as its parameters evolve and compare the results of the modal approach to those of the direct numerical simulation of the partial differential equations. For this aim, we use the Lyapunov exponent. The results here-

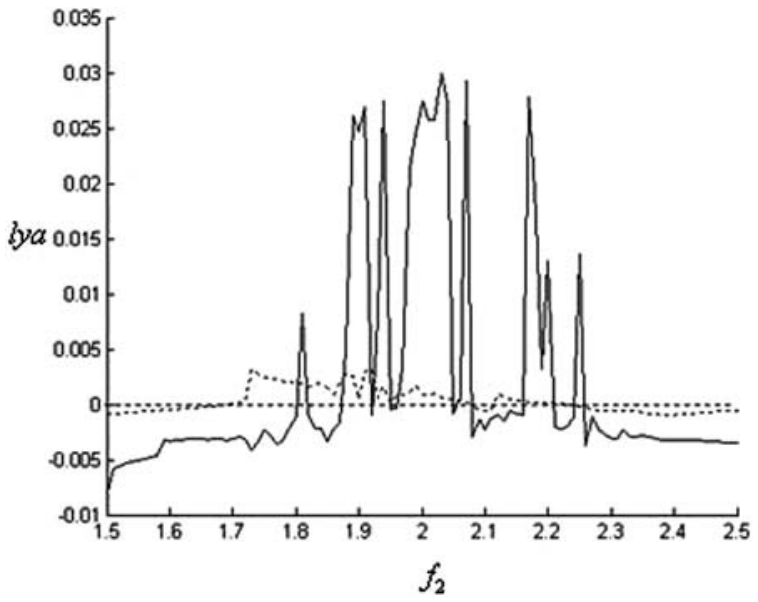

Fig. 4 Variation of the Lyapunov exponent as function of the coupling coefficient $f_{2}$ from the modal approach (lines) and from the finite difference simulation (dash line) with $b=0.1$; $a=\frac{1}{k_{1}^{2}} ; w_{01}=w_{0}=1 ; \varepsilon_{1}=2.466 ; f_{1}=3.518$

after are obtained by numerically solving (16) and (17) and (18) and (19) with their corresponding variational equations. In the case of finite difference simulation, the Lyapunov exponent is defined by

lyan $=\lim _{t \rightarrow \infty} \frac{\ln \left(d_{1}(t)\right)}{t}$

with

$d_{1}=\sqrt{d q^{2}+\left(\frac{d}{d t} d q\right)^{2}+\sum_{i=1}^{n} d v_{i}^{2}+\sum_{i=1}^{n}\left(\frac{\partial}{\partial t} d v_{i}\right)^{2}}$

while for the ordinary differential equations (see (16) and (17)), one has

lyas $=\lim _{t \rightarrow \infty} \frac{\ln \left(d_{2}(t)\right)}{t}$

with

$d_{2}=\sqrt{d q^{2}+\left(\frac{d}{d t} d q\right)^{2}+d y_{1}^{2}+\left(\frac{d}{d t} d y_{1}\right)^{2}}$

where $d q, d v_{i}$ and $d y_{1}$ are the variation of $q, v_{i}$ and $y_{1}$, respectively.

Figure 4 shows the Lyapunov exponent as the coupling coefficient $f_{2}$ increases. One finds that for $f_{2} \in[1.85 ; 2.3]$, there is a series of domain corresponding to a chaotic dynamics with the modal approach while with the finite difference scheme, this 


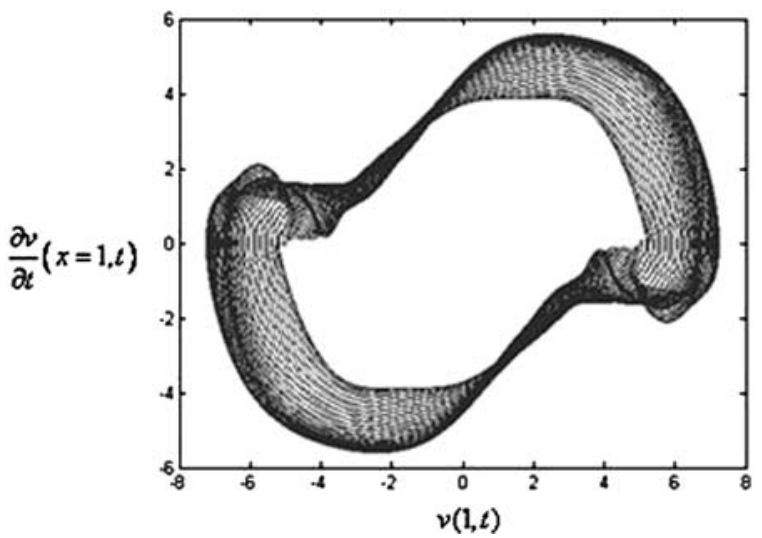

(a)

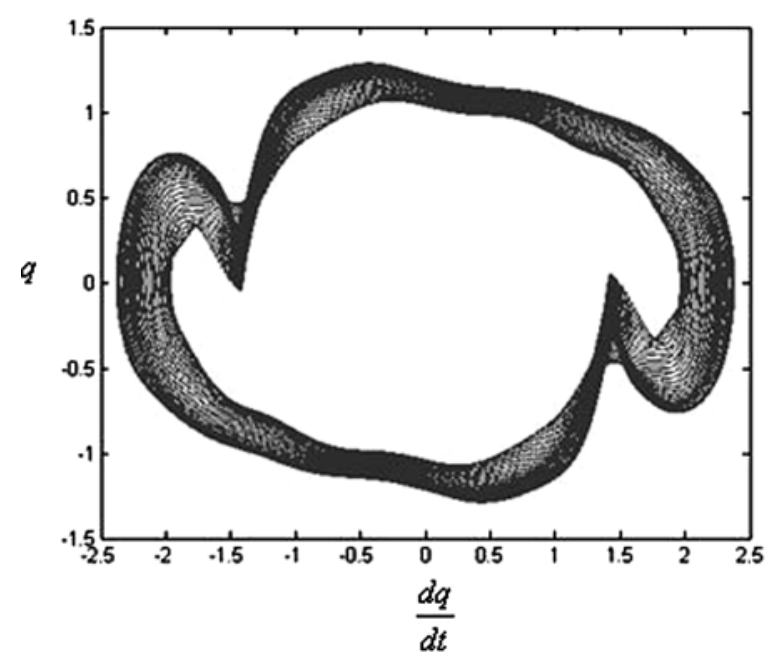

(b)

Fig. 5 Phase portrait of the mechanical part (a) and electric part (b) from the finite difference simulation with the parameters of Fig. 4 and $f_{2}=2.2$

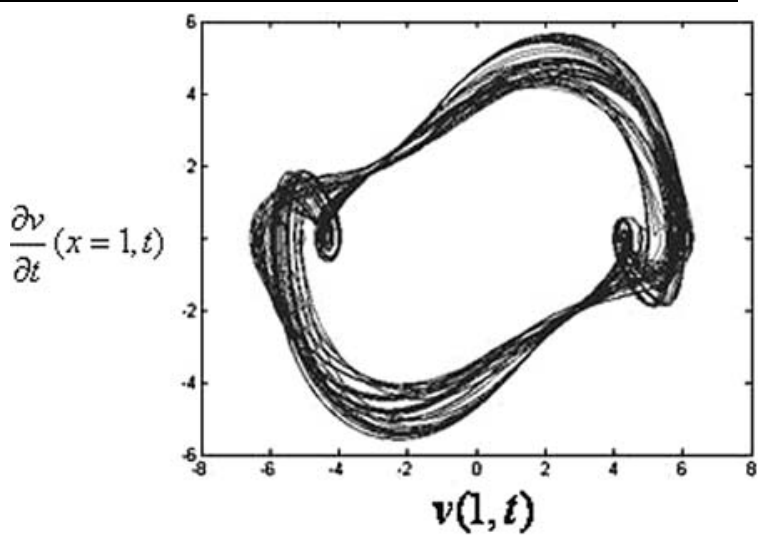

(a)

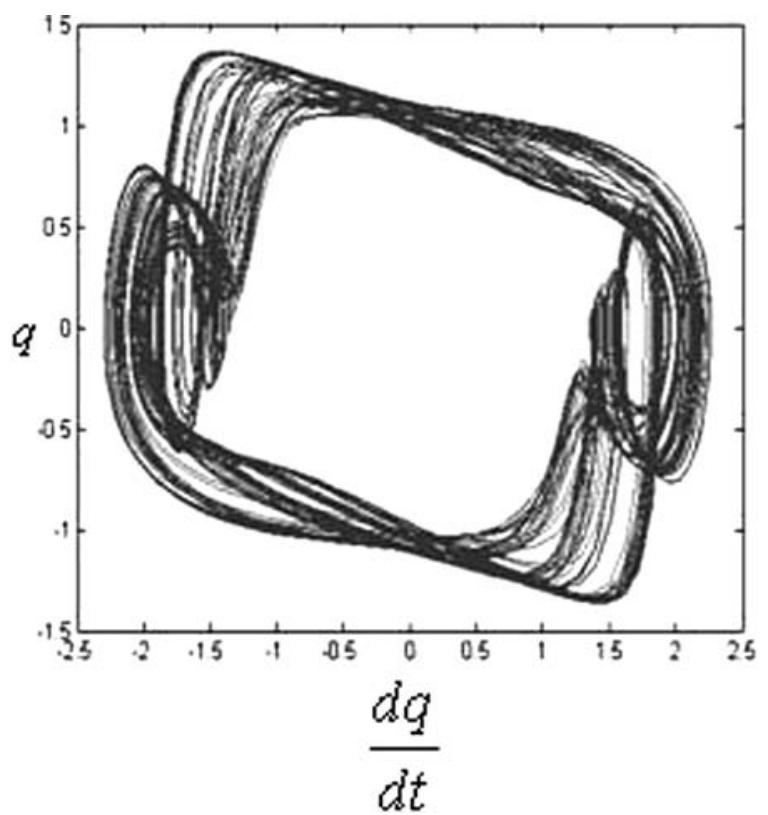

(b)

Fig. 6 Phase portrait of the mechanical part (a) and electric part (b) from modal approach with the parameters of Fig. 4 and $f_{2}=2.2$

only one mode of vibration. Although at resonance, the first mode possesses the main part of the energy of the system, the effects of the neglected modes can be perceptible on the sensitive behaviors as found in the chaotic state.

The next section is devoted to the synchronization of the regular and chaotic states of two electromechanical devices. 
Fig. 7 The Master-slave electromechanical devices

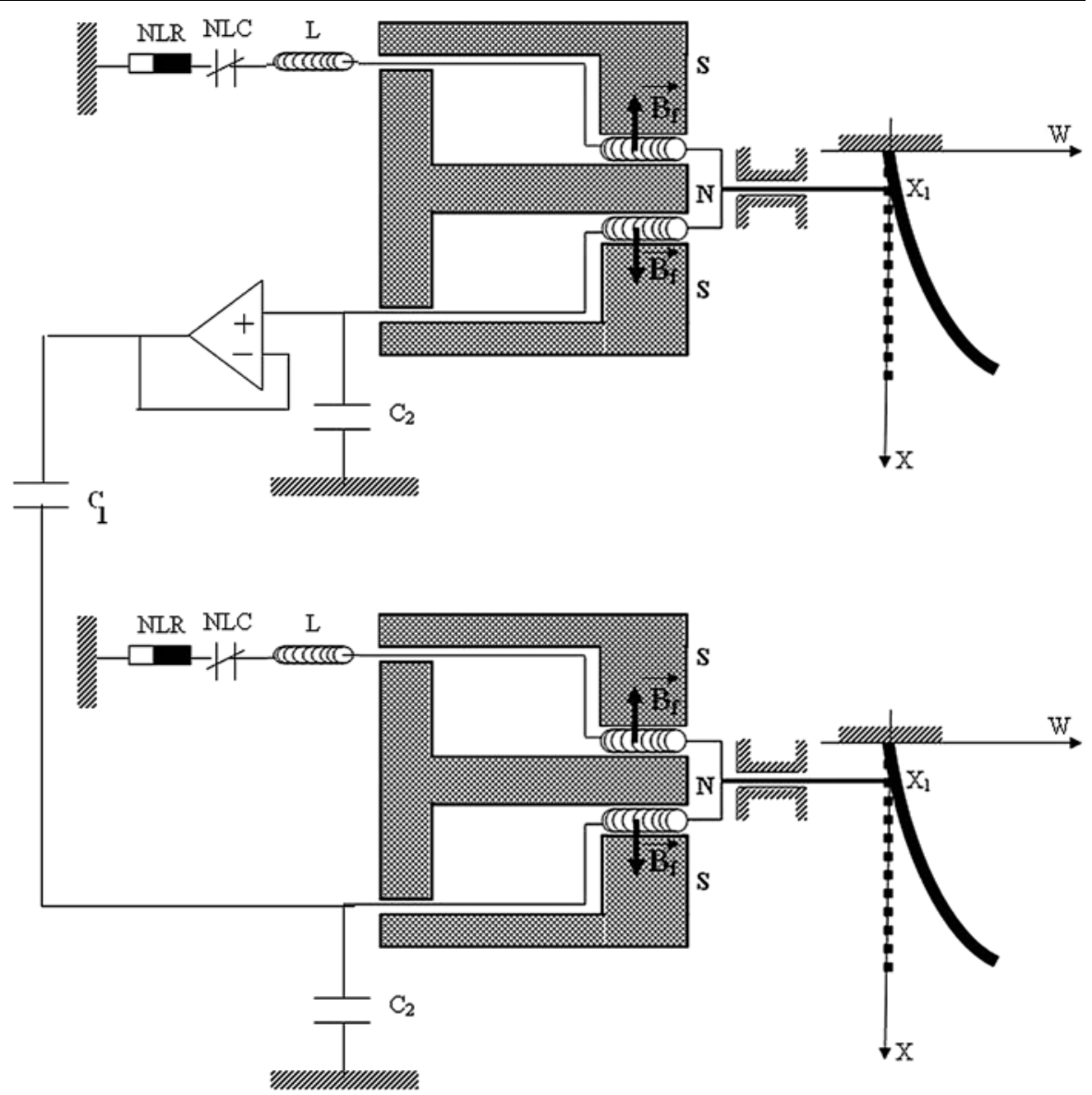

\section{Synchronization of two self-sustained electromechanical systems with flexible arm}

As we noted in the introduction, synchronization is of crucial importance in automation engineering where devices working in an ordered way are required. The work dynamics may be periodic or chaotic depending on the goals and applications consisting, for instance of cutting, drilling, shaking and mixing. The particularity of the devices analyzed here is that if they are started with different initial conditions, they will circulate in the same orbit but with different phase (case of periodic or limit cycle state) or on different complex orbits (case of chaotic behavior). In this section, we deal with the determination of synchronization conditions for two such devices coupled in the masterslave scheme. The analytical analysis, which is complemented by numerical simulation, uses the Floquet theory on the variational equations of the deviation of the slave orbit from the orbit of the master device.

\subsection{Model and equations of motion}

In this section, we derive the characteristics of the unidirectional synchronization of two self-sustained electromechanical devices with flexible arm. The master system is described by the components $q$ and $v$, while the slave system has the corresponding components $p$ and $u$. The enslavement is carried out by an electric device consisting of operational amplifiers (see Fig. 7). The equations of the slave are

$$
\begin{aligned}
& \frac{d^{2} p}{d t^{2}}-\varepsilon_{1}\left(1-\left(\frac{d p}{d t}\right)^{2}\right) \frac{d p}{d t}+w_{0}^{2} p+b p^{3} \\
& \quad+f_{2} \frac{\partial u}{\partial t} \delta\left(x-x_{1}\right) \\
& \quad+K(p-q) H\left(t-T_{0}\right)=0 \\
& \frac{\partial^{2} u}{\partial t^{2}}+\varepsilon_{2} \frac{\partial u}{\partial t}+a \frac{\partial^{4} u}{\partial x^{4}}-f_{1} \frac{d p}{d t} \delta\left(x-x_{1}\right)=0 .
\end{aligned}
$$


In the modal approach, they transform themselves to

$$
\begin{aligned}
& \frac{d^{2} p}{d t^{2}}-\varepsilon_{1}\left(1-\left(\frac{d p}{d t}\right)^{2}\right) \frac{d p}{d t}+w_{0}^{2} p+b p^{3}+f_{21} \frac{d \Upsilon_{1}}{d t} \\
& \quad+K(p-q) H\left(t-T_{0}\right)=0 \\
& \frac{d^{2} \Upsilon_{1}}{d t^{2}}+\varepsilon_{2} \frac{d \Upsilon_{1}}{d t}+w_{01}^{2} \Upsilon_{1}-f_{11} \frac{d p}{d t}=0
\end{aligned}
$$

where $K=\frac{C_{1}}{C_{2}\left(C_{1}+C_{2}\right) L \omega_{1}}$ (with $C_{0} \gg C_{2}$ ), is the dimensionless feedback coupling coefficient or strength, $H(x)$ the Heaviside function defined as $H(x)=0$ for $x<0$ and $H(x)=1$ for $x>0$, and $T_{0}$ the onset time of synchronization.

\subsection{The formalism for optimal synchronization}

When the synchronization process is launched, the slave configuration changes and one would like to determine the range of $K$ for the synchronization to be achieved, and for the dynamics of the slave to remain stable. To carry out such an investigation, let us introduce the following variables $\zeta=p-q$ and $z=u-v$ which measure the nearness of the slave to the master. Introducing these variables in (35) and (36) and taking $z(x, t)=\eta_{1}(t) \phi_{1}(x)$, we obtain that $\zeta$ and $\eta_{1}$ satisfy the variational equations

$$
\begin{aligned}
& \frac{d^{2} \varsigma}{d t^{2}}-\varepsilon_{1}\left(1-3\left(\frac{d q}{d t}\right)^{2}\right) \frac{d \varsigma}{d t} \\
& \quad+\Omega^{2} \varsigma+f_{21} \frac{d \eta_{1}}{d t}=0 \\
& \frac{d^{2} \eta_{1}}{d t^{2}}+\varepsilon_{2} \frac{d \eta_{1}}{d t}+\omega_{01}^{2} \eta_{1}-f_{11} \frac{d \varsigma}{d t}=0
\end{aligned}
$$

where $\Omega^{2}=w_{0}^{2}+3 b q^{2}+K$

The synchronization process is achieved when $\zeta$ and $z$ go to zero as $t$ increases or, practically are less than a given precision. The behavior of the slave depends on $K$ and the form of the master. Assuming that $\varepsilon_{1}$ is small, the master variables take the form

$q=A \cos \left(\omega_{0} t-\varphi_{1}\right)$,

$y_{1}=B \cos \left(\omega_{01} t-\varphi_{2}\right)$

where the amplitudes $A$ and $B$ depend on the system parameters as described by (26) and (27). With this form of the master, (37) and (38) takes the form

$\frac{d^{2} \zeta}{d t^{2}}+F_{1}(t) \frac{d \zeta}{d t}+G_{1}(t) \zeta+f_{21} \frac{d \eta_{1}}{d t}=0$, $\frac{d^{2} \eta_{1}}{d t^{2}}+F_{2}(t) \frac{d \eta_{1}}{d t}+G_{2}(t) \eta_{1}-f_{11} \frac{d \zeta}{d t}=0$

with $F_{1}(t)=\lambda_{0}-\frac{3}{2} A^{2} \omega_{0}^{2} \varepsilon_{1} \cos (2 \xi), G_{1}(t)=\Omega^{2}$, $\lambda_{0}=\varepsilon_{1}\left(-1+\frac{3}{2} A^{2} \omega_{0}^{2}\right), F_{1}(t)=\varepsilon_{2}, G_{2}(t)=\omega_{01}^{2}, \xi=$ $\omega_{0} t-\gamma_{1}$.

Setting the following transformations

$$
\begin{aligned}
& \zeta=U \exp \left(-\frac{1}{2} \int F_{1}(t) d t\right), \\
& \eta_{1}=V \exp \left(-\frac{1}{2} \int F_{2}(t) d t\right)
\end{aligned}
$$

we rewrite (41) and (42) in the standard form

$$
\begin{aligned}
& \frac{d^{2} U}{d t^{2}}+F(t) U \\
& +f_{21}\left(\frac{d V}{d t}-G_{2}(t) V\right) \exp (\psi)=0, \\
& \frac{d^{2} V}{d t^{2}}+G(t) V \\
& \quad+\left(R(t) U-f_{11} \frac{d U}{d t}\right) \exp (-\psi)=0
\end{aligned}
$$

with

$$
\begin{aligned}
F(t)= & \delta_{11}+2 \epsilon_{11} \sin (2 \xi)+2 \epsilon_{12} \cos (2 \xi) \\
& +2 \epsilon_{13} \cos (4 \xi), \\
G(t)= & \delta_{21}, \\
R(t)= & \delta_{22}+2 \epsilon_{21} \cos (2 \xi), \\
\psi=- & \frac{1}{2}\left(\varepsilon_{2}-\lambda_{0}\right) t+\frac{3}{8} A^{2} w \varepsilon_{1} \sin (2 \xi), \\
\delta_{11}= & \Omega^{2}-\frac{\lambda_{0}^{2}}{4}-\frac{9}{32} A^{4} w^{4} \varepsilon_{1}, \\
\epsilon_{11}= & -\frac{3}{4} A^{4} w^{3} \varepsilon_{1}, \\
\epsilon_{12}= & \frac{3}{4} b A^{2}+\frac{3}{8} A^{2} w^{2} \lambda_{0} \varepsilon_{1}, \\
\epsilon_{13}= & -\frac{9}{64} A^{4} w^{4} \varepsilon_{1}^{2}, \quad \delta_{22}=\frac{\lambda_{0} f_{11}}{2}, \\
\epsilon_{21}= & -\frac{3}{8} A^{2} w^{2} \varepsilon_{1} f_{11}, \quad \delta_{21}=w_{01}^{2}-\frac{\varepsilon_{2}^{2}}{4} .
\end{aligned}
$$


Equations (45) and (46) are two coupled Hill's equations. According to the Floquet theory $[18,19]$, the solutions are

$U=\alpha(t) \exp \left(\theta_{1} t\right)=\sum_{n=-\infty}^{n=+\infty} \alpha_{n} \exp \left(a_{n} t\right)$,

$V=\beta(t) \exp \left(\theta_{2} t\right)=\sum_{n=-\infty}^{n=+\infty} \beta_{n} \exp \left(b_{n} t\right)$

where $a_{n}=\theta_{1}+2 J n \omega_{0}, b_{n}=\theta_{2}+2 J n \omega_{0}\left(J^{2}=-1\right)$. The function $\alpha(t)=\alpha(t+\pi)$ and $\beta(t)=\beta(t+\pi)$ are replaced by the Fourier series, with $\theta_{1}, \theta_{2} \in \mathbf{C}$ and $\alpha_{n}, \beta_{n} \in \mathbf{R}$. Inserting (47) and (48) into (45) and (46) yields $(\forall n \in \mathbf{N})$

$$
\begin{aligned}
& \sum_{n=-\infty}^{n=+\infty} e^{2 J n \omega_{0}}\left\{\alpha_{n}\left(a_{n}^{2}+\delta_{11}\right)+\alpha_{n+1}\left(\epsilon_{12}+J \in_{11}\right) e^{\psi_{2}}\right. \\
& \quad+\alpha_{n-1}\left(\epsilon_{12}-J \epsilon_{11}\right) e^{-\psi_{1}}+\alpha_{n+2} \in_{13} e^{-2 \psi_{1}} \\
& \quad+\alpha_{n-2} \in_{13} e^{2 \psi_{2}} \\
& \left.\quad+f_{21} \beta_{n}\left(b_{n}-\frac{\epsilon_{2}}{2}\right) e^{v}\right\}=0
\end{aligned}
$$

$$
\begin{aligned}
& \sum_{n=-\infty}^{n=+\infty} e^{2 J n \omega_{0}}\left\{\alpha_{n}\left(-f_{11} a_{n}+\delta_{22}\right) e^{-v}\right. \\
& \quad+\alpha_{n+1} \in_{21} e^{\psi_{2}-v}+\alpha_{n-1} \epsilon_{21} e^{-\psi_{1}-v} \\
& \left.\quad+\beta_{n}\left(b_{n}^{2}+\delta_{21}\right)\right\}=0
\end{aligned}
$$

Equating each of the coefficients of the exponential functions to zero, one obtains the following infinite set $(S)$ of linear, algebraic and homogeneous equations for the $\alpha_{n}$ and $\beta_{n}$

$$
\left\{\begin{array}{l}
\alpha_{n}\left(a_{n}^{2}+\delta_{11}\right)+\alpha_{n+1}\left(\epsilon_{12}+J \in_{11}\right) e^{\psi_{2}} \\
\quad+\alpha_{n-1}\left(\epsilon_{12}-J \in_{11}\right) e^{-\psi_{1}} \\
\quad+\alpha_{n+2} \in_{13} e^{-2 \psi_{1}} \\
\quad+\alpha_{n-2} \in_{13} e^{2 \psi_{2}}+f_{21} \beta_{n}\left(b_{n}-\frac{\epsilon_{2}}{2}\right) e^{\psi}=0 \\
\alpha_{n}\left(-f_{11} a_{n}+\delta_{22}\right) e^{-\psi}+\alpha_{n+1} \in_{21} e^{\psi_{2}-\psi} \\
\quad+\alpha_{n-1} \epsilon_{21} e^{-\psi_{1}-\psi}+\beta_{n}\left(b_{n}^{2}+\delta_{21}\right)=0
\end{array}\right.
$$

where $v=\left(\theta_{2}-\frac{\varepsilon_{2}}{2}\right) t-\left(\theta_{1}-\frac{\lambda_{0}}{2}\right) t+\frac{3}{8} A^{2} \omega_{0} \varepsilon_{1} \sin (2 \xi)$, $\psi_{1}=2 J \gamma_{1}+\theta_{1} t, \psi_{2}=2 J \gamma_{1}-\theta_{2} t$. Applying the con- sideration of [2], we find that the boundary that separates the stability from the instability domains, is given by

$\operatorname{det}(S)=0$

Here we limit the calculation to the sixth order Hill's determinant of the algebraic system $(S)$. Since, we have

$$
\zeta=\exp \left\{\left(\theta_{1}-\frac{\lambda_{0}}{2}\right) t-\frac{3}{8} \epsilon_{1} w A^{2} \sin (2 \xi)\right\} \alpha(t),
$$

$\eta_{1}=\exp \left\{\left(\theta_{2}-\frac{\epsilon_{2}}{2}\right) t\right\} \beta(t)$.

The Floquet theory states that the transition from stability to instability domains (or the reverse) occurs only in the two following conditions:

- $\pi$-periodic transitions at $\theta_{1}=\theta_{1}^{1}=\frac{\lambda_{0}}{2}$ and $\theta_{2}=$ $\theta_{2}^{1}=\frac{\epsilon_{2}}{2}$

- $2 \pi$-periodic transitions at $\theta_{1}=\theta_{1}^{2}=\frac{\lambda_{0}}{2}+J$ and $\theta_{2}=\theta_{2}^{2}=\frac{\epsilon_{2}}{2}+J$.

Thus, replacing $\theta_{k}$ by $\theta_{k}^{k}(k=1,2)$ in (52), we obtain an equation which helps us to determine the range of $K$ in which the synchronization process is stable.

\subsection{Synchronization of the oscillatory dynamics}

In this subsection, we consider the master and the slave systems with a periodic behavior and compare the results of numerical simulation of (33) and (34) and (35) and (36) to that of the above analytical treatment. The amplitude $A=0.31$ is obtained from (26) and (27) with $\varepsilon_{2}=0.01$ while the frequency $\omega_{0}$ is set equal to $\omega_{01}$ (at the resonance). From (52), the stability is achieved for $K \in]-11.36 ; 0] \cup] 0 ;+\infty]$ with the parameters of Fig. 2. For the numerical simulation of (33) and (34) and (35) and (36) along with (9) and (10) and (16) and (17) of the master, we use the initial conditions $\left(q, \frac{d q}{d t}, v, \frac{\partial v}{\partial t}\right)=$ $(5.0,5.0,0.0,0.0)$ for the master and $\left(p, \frac{d p}{d t}, u, \frac{\partial u}{\partial t}\right)=$ $(4.0,4.0,0.0,0.0)$ for the slave. We obtain that the synchronization domain is $K \in]-12.4 ; 0] \cup] 0 ;+\infty$ ] from the modal approach (ordinary differential equations) and $K \in]-12.6 ; 0] \cup] 0 ;+\infty]$ from the direct numerical simulation of the partial differential equations. We take $T_{0}=800$ and assume that the synchronization is achieved when $|q-p|<h_{0}, \forall t>$ 


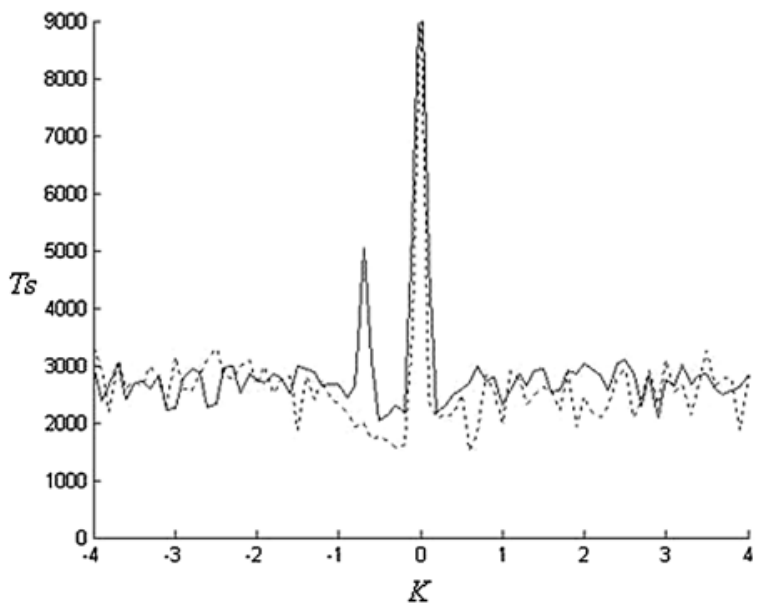

Fig. 8 Synchronization time $T_{s}$ versus $K$ with the parameter of Fig. 2 and $\varepsilon_{2}=0.01$ from the finite difference simulation (dash line) and the modal approach (line)

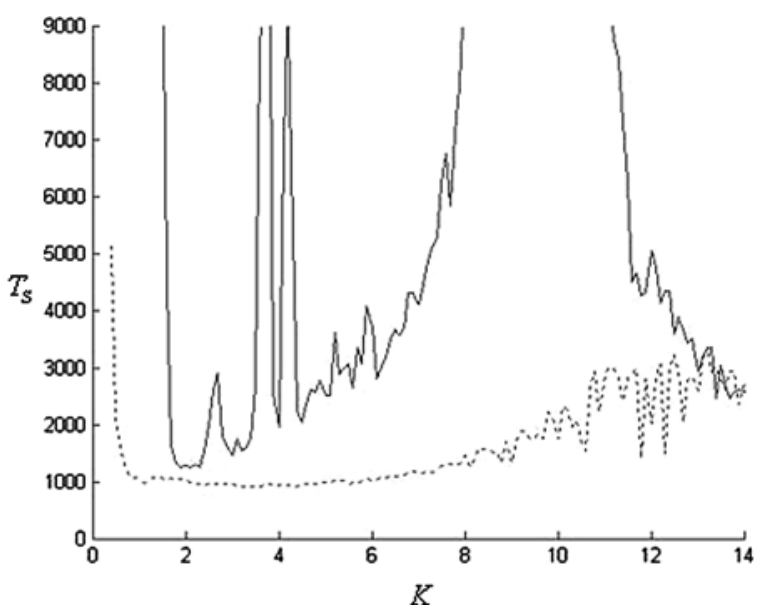

Fig. 9 Synchronization time $T_{s}$ versus $K$ with the parameter of Fig. 5 from the finite difference simulation (dash line) and the modal approach (line) in the chaotic regime

$T_{0}$ with $h_{0}=10^{-10}$. Figure 8 shows the synchronization time $T_{S}$ versus $K$. The agreement between the two approaches and the analytical investigation is quite acceptable. The singularity at $K=-0.7$ can be the signature of parametric resonances.

\subsection{Case of chaotic states}

Hereafter, the master and slave systems are in the chaotic state. We proceed to numerical simulation of (33-34) and (35-36) to determine the range of $K$ for which the synchronization is achieved. The criterion

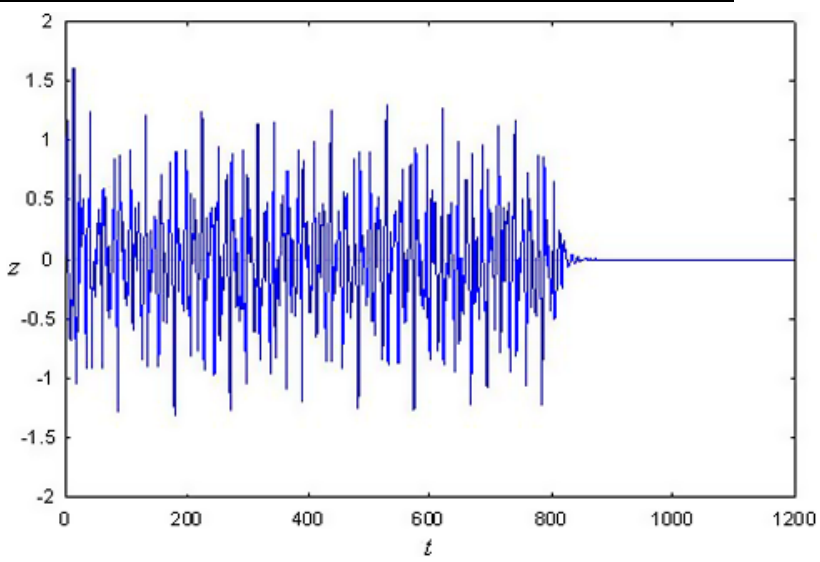

(a)

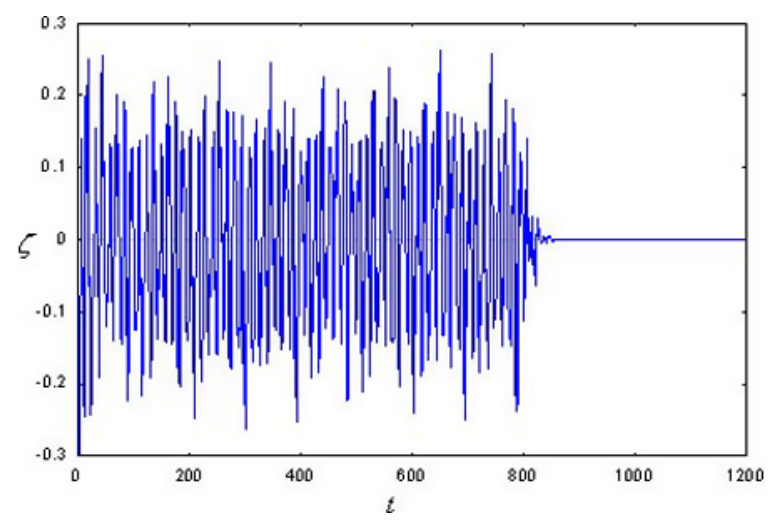

(b)

Fig. 10 Time history of the deviations $z$ (a) and $\zeta$ (b) with the parameters of Fig. 5 and $K=3$ from finite difference simulation: case of synchronization failure

of numerical synchronization is that used for the regular state. The initial conditions are $\left(q, \frac{d q}{d t}, v, \frac{\partial v}{\partial t}\right)=$ $(3.5,3.2,0.0,0.0)$ for the master and $\left(p, \frac{d p}{d t}, u, \frac{\partial u}{\partial t}\right)=$ $(4.0,4.0,0.0,0.0)$ for the slave. We vary $K$ between -15 and +15 to find the synchronization domains. For the modal approach, we find that the synchronization is achieved for $K \in] 1.5 ; 3.7] \cup] 3.8 ; 4.2] \cup] 4.2 ; 8] \cup$ ] $11 ; 15]$, while the finite differences simulation gives the synchronization for $K \in] 0.4 ; 15]$. The synchronization time $T_{s}$ is plotted versus $K$ and the results are reported in Fig. 9 for the two approaches. The difference between the modal approach and the finite difference simulation is very important if compared to what is observed in the case of oscillatory behavior. This is understandable since the harmonic oscillatory approximation (see (39) and (40)) used for the formalism is invalid here. Indeed, it cannot approximate the time 


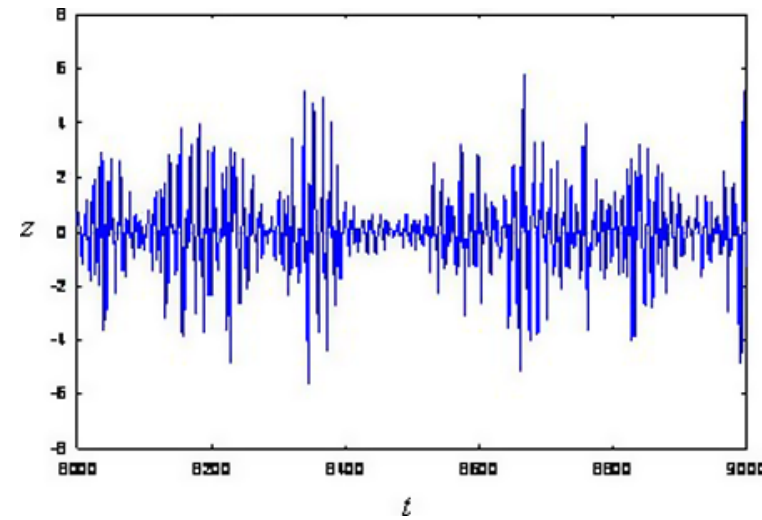

(a)

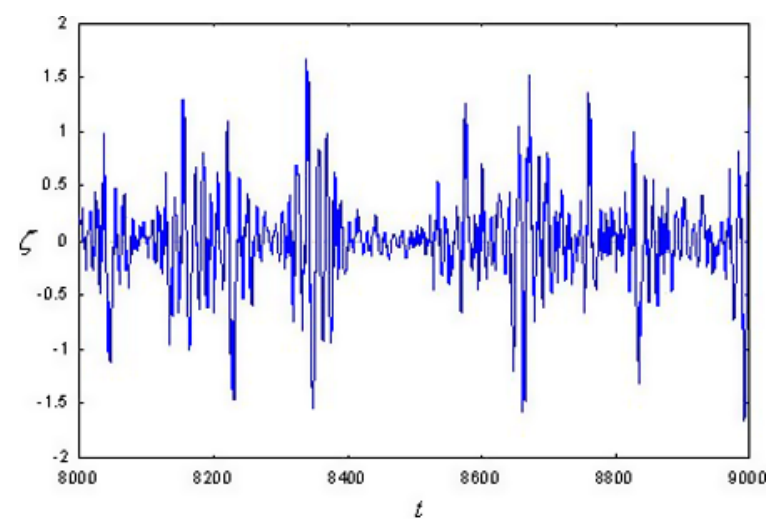

(b)

Fig. 11 Time history of the deviations $z$ (a) and $\zeta$ (b) with the parameters of Fig. 5 and $K=-1$ form the finite difference simulation: case of synchronization

behavior of the chaotic state. Figures 10 and 11 show, respectively, the deviation between the slave and the master in the case of synchronization, and in the case where the synchronization process has failed.

\section{Conclusion}

This paper has dealt with the dynamics, chaos and synchronization of self-sustained electromechanical systems with flexible arm consisting of a RayleighDuffing oscillator coupled magnetically to a flexible beam. The averaging method has been used to determine the amplitudes of the oscillatory behavior. The Lyapunov exponent helps us to study the chaotic behavior and typical chaotic phase portraits were reported. For the synchronization process, the analytical investigation has been based on the properties of the
Hill equation which describes the deviation between the slave and the master devices. The analytical results have been compared to those of the semi-analytical studies as well as to those of a direct numerical simulation of the partial differential equations. The next step following this study is to carry out experimental investigations where the effects of a parameters mismatch is unavoidable.

Acknowledgements This work is supported by the Academy of Sciences for Developing World (TWAS) under Research Grant N. 03-322 RG/PHYS/AF/AC.

P. Woafo acknowledges the support from the Humboldt Foundation and the Department of Nonlinear Dynamics, MaxPlanck Institute for Dynamics and Self-Organisation (Gottingen, Germany).

\section{References}

1. Chedjou, J.C., Woafo, P., Domngang, S.: Shilnikov chaos and dynamics of a self-sustained electromechanical transducer. ASME J. Vib. Acoust. 123, 170-174 (2001)

2. Yamapi, R., Woafo, P.: Dynamics and synchronization of self-sustained electromechanical devices. J. Sound Vib. 285, 1151-1170 (2005)

3. Kitio Kwuimy, C.A., Woafo, P.: Dynamics of a selfsustained electromechanical system with flexible arm and cubic coupling. Commun. Nonlinear Sci. Numer. Simul. 12, 1504-1517 (2007)

4. Jerrelind, J., Stensson, A.: Nonlinear dynamics of parts in engineering systems. Chaos Solitons Fractals 11, 24132428 (2000)

5. Chembo Kouomou, Y., Woafo, P.: Triple resonant states and chaos control in an electrostatic transducer with two outputs. J. Sound Vib. 270, 75-92 (2004)

6. Luo, A.C.J., Wang, F.Y.: Nonlinear dynamics of a microelectromechanical system with time-varying capacitors. ASME J. Vib. Acoust. 126, 77-83 (2004)

7. Yamapi, R., Moukam Kakmeni, F.M., Chabi Orou, J.B.: Nonlinear dynamics and synchonization of coupled electromechanical systems with multiple functions. Commun. Nonlinear Sci. Numer. Simul. 12, 543-567 (2007)

8. Yamapi, R., Woafo, P.: Synchronized states in a ring of four mutually coupled self-sustained electromechanical devices. Commun. Nonlinear Sci. Numer. Simul. 11, 186-202 (2006)

9. Raskin, J.P., Brown, A.R., Khuri-Yakub, B.T., Rebeiz, G.M.: A novel parameter MEMS amplifier. J. Microelectromech. Syst. 9, 528-537 (2000)

10. Teufel, A., Steindl, A., Troger, H.: Synchronization of two flow excited pendula. Commun. Nonlinear Sci. Numer. Simul. 11, 577-594 (2006)

11. Bowong, S.: Adaptive synchronization between two different chaotic dynamical systems. Commun. Nonlinear Sci. Numer. Simul. 12, 976-985 (2007)

12. Dibakar, G., Papri, S., Roy Chowdhury, A.: On synchronization of a forced delay dynamical system via the Galerkin approximation. Commun. Nonlinear Sci. Numer. Simul. 12, 928-941 (2007) 
13. Dibakar, G., Roy Chowdhury, A., Papri, S.: On the various kinds of synchronization in delayed Duffing-Van der Pol system. Commun. Nonlinear Sci. Numer. Simul. 13, 790803 (2008)

14. Oksasoglu, A., Vavriv, D.: Interaction of low and highfrequency oscillations in a nonlinear RLC circuit. IEEE Trans. Circuits Syst. 41, 669-672 (1994)

15. Hasler, M.J.: Electrical circuit with chaotic behaviour. Proc. IEEE 75, 1009-1021 (1987)
16. King, G.P., Gaito, S.T.: Bistable chaos, I, unfolding the cusp. Phys. Rev. A 46, 3092-3099 (1992)

17. Timoshenko, S., Gere, J.M.: Theory of Elastic Stability, 2nd edn. McGraw-Hill, New York (1961)

18. Nayfeh, A.H., Mook, D.T.: Nonlinear Oscillations. Wiley, New York (1979)

19. Hayashi, C.: Nonlinear Oscillations in Physical Systems. McGraw-Hill, New York (1964) 